\title{
Dimensionality reduction for hand-independent dexterous robotic grasping
}

\author{
Matei Ciocarlie Corey Goldfeder Peter Allen
}

\begin{abstract}
In this paper, we build upon recent advances in neuroscience research which have shown that control of the human hand during grasping is dominated by movement in a configuration space of highly reduced dimensionality. We extend this concept to robotic hands and show how a similar dimensionality reduction can be defined for a number of different hand models. This framework can be used to derive planning algorithms that produce stable grasps even for highly complex hand designs. Furthermore, it offers a unified approach for controlling different hands, even if the kinematic structures of the models are significantly different. We illustrate these concepts by building a comprehensive grasp planner that can be used on a large variety of robotic hands under various constraints.
\end{abstract}

\section{INTRODUCTION AND RELATED WORK}

One of the hardest problems in robotic grasping is the creation of control algorithms for new hand designs that are beginning to rival the human hand in complexity. Researchers studying robotic grasping have struggled to at least partially replicate human versatility when designing artificial counterparts. However, if we wish to reproduce human-like grasping it would seems natural to draw inspiration not only from the hardware of the human hand, but also from the software; that is, the way the hand is controlled by the brain. This may initially sound like an overly lofty goal: a large part of the human cortex is dedicated to grasping and manipulation, and it would seem reasonable to assume that all of this cognitive machinery is dedicated to finely controlling individual joints and generating highly flexible hand postures. However, results in both robotics and neuroscience research point to the contrary, emphasizing that a majority of the human hand control during common grasping tasks lacks individuation in finger movements.

Attempts to formalize human tendency to simplify the space of possible grasps can be traced back to Napier's pioneering grasp taxonomy [1], updated later by Cutkosky [2]. In [3], Iberall reviewed a large field of work on grasp taxonomies, from areas such as anthropology, medical, rehabilitation and robotics. These studies suggest that, while the configuration space of dexterous hands is high-dimensional and very difficult to search directly, most useful grasps can be found in the vicinity of a small number of discrete points.

Santello et al. [4] tested this hypothesis by using a Cyberglove to record joint angle information from human

This work was funded in part by NIH BRP grant 1RO1 NS 050256-01A2 and a National Defense Science and Engineering Graduate Fellowship.

All authors are with the Department of Computer Science, Columbia University, NY 10027, USA

E-mail: $\{$ cmatei, coreyg, allen\}ecs.columbia.edu subjects during grasping tasks. Their results suggest that a continuous subspace, rather then a finite number of discrete postures, can be used to approximate human hand control during grasping. We will expand upon this topic later in this paper. Peters and Jenkins [5] compared a number of dimensionality reduction techniques used to extract relevant two-dimensional manifolds from datasets recorded from human operators. Tsoli and Jenkins [6] showed that, once such a manifold is obtained, two dimensional control is sufficient for teleoperation involving tasks such as power and precision grasps and tapping motion.

The body of work exploring human hand control has also been applied to the problem of automatic grasp planning. Miller et al. [7] used the grasp taxonomy concept to define a number of starting positions, or pre-grasps, when searching for good grasps of a given object using a robotic hand. Aleotti and Caselli [8] used a Cyberglove to record grasp trajectories and postures used by test subjects on a set of example objects and replicated those trajectories on the same objects using NURBS. Li and Pollard [9] used a shape matching approach, sampling an object into a dense cloud of oriented points and matching against a small database of known human hand poses. For a general review of the robotic grasp planning problem we also refer the reader to [10], [11].

In the work presented in this paper, we build upon Santello's analysis of human hand motion during grasping to produce control algorithms for robotic hands. We show how using this approach reduces the dimensionality of the problem to the point where it is reasonable to search the hand configuration space for good grasps of a given object. The resulting grasp planner can be applied to a number of different hand designs, including a model of the human hand.

\section{EIGENGRASPS}

Any hand posture is fully specified by its joint values, and can therefore be thought of as a point in a high-dimensional joint space. If $d$ is the number of degrees of freedom (DOF) of the hand, than a posture $\boldsymbol{p}$ can be defined as

$$
\boldsymbol{p}=\left[\begin{array}{llll}
\theta_{1} & \theta_{2} & \ldots & \theta_{d}
\end{array}\right] \in \mathcal{R}^{d}
$$

where $\theta_{i}$ is the value of $i$-th degree of freedom.

As we have discussed in our literature review, previous research suggests that most human grasping postures derive from a relatively small set of discrete pregrasp shapes. This would imply that the range of postures used in everyday grasping tasks will exhibit significant clustering in the $d$ dimensional DOF space. Santello et al. [4] verified this hypotheses by collecting a large set of data containing grasping 
poses from subjects that were asked to shape their hands as if they were grasping a familiar object. Principal Component Analysis of this data revealed that the first two principal components account for more than $80 \%$ of the variance, suggesting that a very good characterization of the recorded data can be obtained using a much lower dimensionality approximation of the joint space.

In our work, we will refer to the Principal Components of these postures as eigengrasps. The implication is that they form a low-dimensionality basis for grasp postures, and can be linearly combined to closely approximate most common grasping positions. Each eigengrasp $\boldsymbol{e}_{i}$ is a $d$ dimensional vector and can also be thought of as direction of motion in joint space. Motion along one eigengrasp direction will usually imply motion along all (or most) degrees of freedom of the hand.

$$
\boldsymbol{e}_{i}=\left[\begin{array}{llll}
e_{i, 1} & e_{i, 2} & \ldots & e_{i, d}
\end{array}\right]
$$

By choosing a basis comprising $b$ eigengrasps, a hand posture placed in the subspace defined by this basis can be expressed as a function of the amplitudes $a_{i}$ along each eigengrasp direction:

$$
\boldsymbol{p}=\sum_{i=1}^{b} a_{i} \boldsymbol{e}_{i}
$$

and is therefore completely defined by the amplitudes vector $\boldsymbol{a}=\left[\begin{array}{lll}a_{1} & \ldots & a_{b}\end{array}\right] \in \mathcal{R}^{b}$.

\section{A. Effective Degrees of Freedom}

An important question to consider is how many eigengrasps need to be used so that the subspace that they define closely approximates the required range of hand postures. Based on the results of Santello et al. discussed above, we have used the two dominant eigengrasps of the human hand in our work, and will show how they produce good results. It is important to note that our study is primarily concerned with grasp synthesis for common everyday objects and that another choice of eigengrasps might be necessary in a different problem domain such as object manipulation or with another dataset, containing unusually complex or difficult to grasp objects.

An intriguing corollary question is whether the results obtained using such a small set of eigengrasps imply that the other DOF's of the hand are useless. We can provide a number of arguments to the contrary: as shown in [4], eigengrasps 3 through 6 (in decreasing order of importance), while accounting for less than $20 \%$ of the variance in hand posture, do not represent noise and are shown to be related to the object to be grasped. Furthermore, the study presented by Santello et al. was performed in the absence of the real object, as subjects reproduced grasps from memory. This suggests that initial grasp planning stages do indeed take place in a low dimensional space, but during the final stages the shape of the object forces the hand to deviate from eigengrasp space in order to conform to the object surface. From this perspective the space defined through eigengrasps can be seen as a pregrasp or grasp planning space, as we shall expand upon below. Finally, complex manipulation tasks are also likely to require usage of the entire range of motion of the hand.

\section{B. Application for Robotic Hand Models}

Although the work of Santello et al. is centered on the study of the human hand, we have found this approach to be extremely useful for robotic hands as well. In our study, we have applied the eigengrasp concept to a total of 5 hand models: a simple gripper, the Barrett hand, the DLR hand [12], the Robonaut hand [13] and finally a human hand model. All our hand models, as well as the eigengrasps used in each case, are presented in table I.

For the human hand we have chosen eigengrasp directions based on the published results in [4], taking advantage of the fact that they have been derived through rigorous study over a large number of recorded samples. Since such data is not available for robotic hand models, we have derived eigengrasps attempting to define grasp subspaces similar to the one obtained using human hand eigengrasps. In most cases, such decisions could be made based directly on the similarities with the human hand. For example, the MCP and IP joints can be mapped to the proximal and distal joints of robotic fingers. In the case of the Barrett hand, changes in the spread angle DOF were mapped to human finger abduction. While we found our choices to produce good results, the optimal choice of eigengrasps for non-human hands, as well as the choice of which eigengrasps to use for a particular task, are open questions and interesting directions for future research.

The eigengrasp concept allows us to design flexible control algorithms that operate identically across all the presented hand models. The key to our approach is that the eigengrasps encapsulate the kinematic characteristics of each hand design. This enables control algorithms that operate on eigengrasp amplitudes to ignore low-level operations and concentrate on the high-level task. We believe this method to be similar in spirit to certain aspects of human brain operation, with individual function grouped together in control synergies. Another advantage is the significant dimensionality reduction (by as much as a factor of 10 for complex hands) obtained by operating in the reduced basis eigengrasp space as opposed to the full joint space. In the next section we will derive a grasp planning algorithm that makes use of both these concepts.

\section{GRASP PLANNING USING EIGENGRASPS}

In essence, the grasp planning task can be thought of as an optimization problem in a high-dimensional space that describes both hand posture (intrinsic DOF's) and position (extrinsic DOF's). Consider the goal of minimizing an energy function of the form:

$$
E=f(\boldsymbol{p}, \boldsymbol{w})
$$

If $\boldsymbol{d}$ is the number of intrinsic hand DOF's then $\boldsymbol{p} \in \mathcal{R}^{d}$ represents the hand posture and $\boldsymbol{w} \in \mathcal{R}^{6}$ contains the position and orientation of the wrist. 


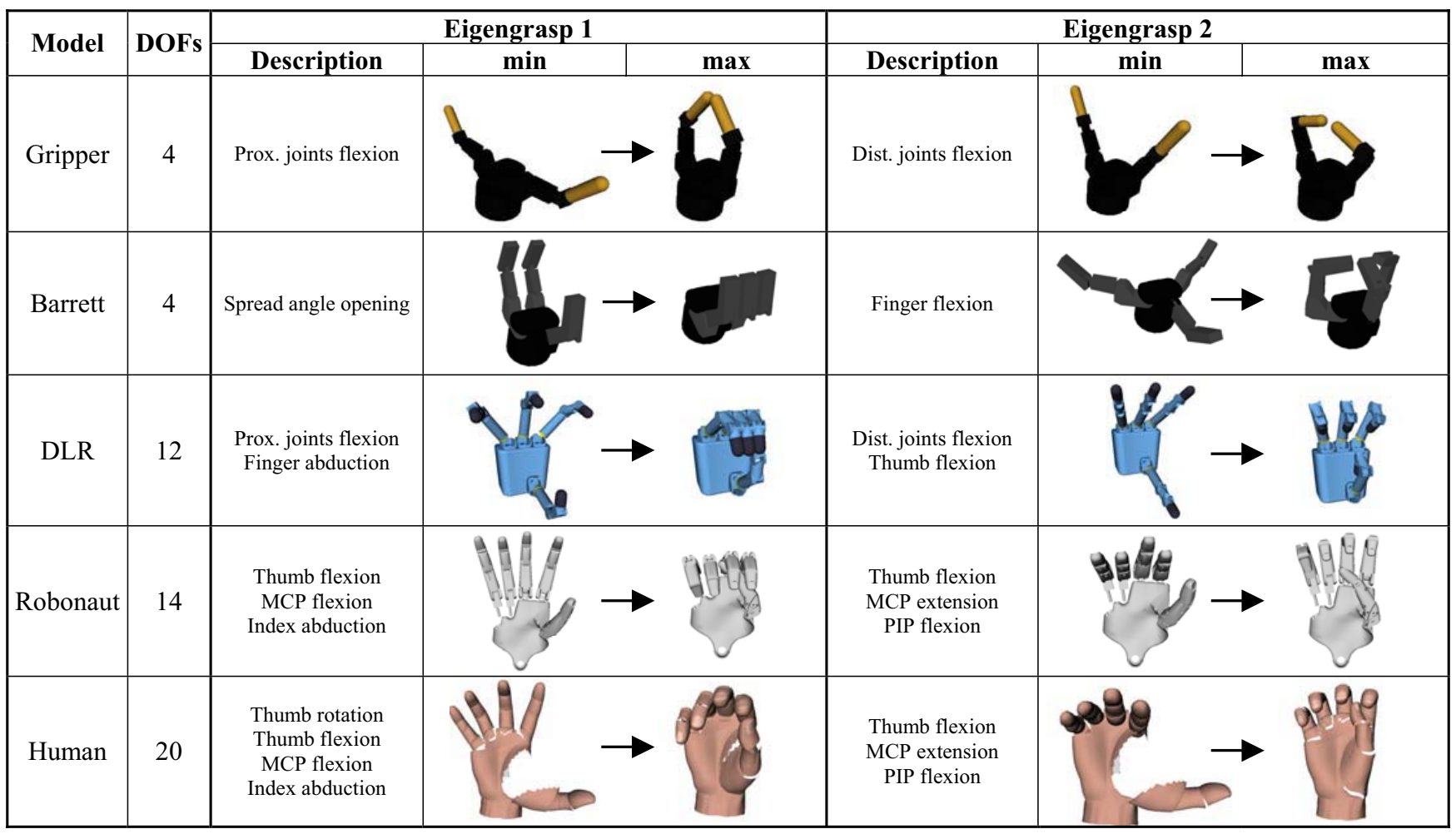

TABLE I

EIGENGRASPS DEFINED FOR THE ROBOTIC HAND MODELS USED IN THIS PAPER.

Intuitively, this energy function has to be related to the quality of the grasp, and we will review a number of possible formulations later in this section. However, most formulations pose a number of problems. First, it can be very difficult, or even impossible, to compute an analytical gradient. Second, such functions are highly non-linear, as small changes in both finger posture and wrist position can drastically alter the quality of the resulting grasp. Finally, the legal parameter space is complex, having to satisfy multiple constraints: prevent inter-penetration with the object to be grasped as well as potential obstacles, and maintain joint values within their acceptable ranges.

\section{A. Optimization Algorithm}

We directly address all of these problems by using simulated annealing as the preferred optimization method (for a general review of the simulated annealing algorithm we refer the reader to [14]). Its stochastic nature makes it a particularly good choice for our task: since new states are generated as random neighbors of the current state, computation of the energy gradient is not necessary, and the algorithm works well on non-linear functions. Furthermore, the possibility of an "uphill move" to a state of higher energy allows it to escape local minima which can trap greedier methods such as gradient descent. However, the random exploration of the input domain means that high dimensionality of the parameter space will severely affect the computational efficiency of this algorithm.

We therefore propose performing the optimization in
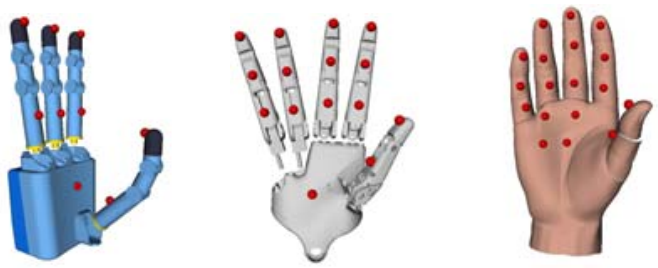

Fig. 1. Desired contact locations for DLR, Robonaut and Human hands

eigengrasp space, as opposed to DOF space. The energy function takes the form

$$
E=f(\boldsymbol{a}, \boldsymbol{w})
$$

where $a \in \mathcal{R}^{2}$ is the vector of eigengrasp amplitudes. This effectively reduces the parameter space to 8 dimensions (2 eigengrasp amplitudes plus 6 extrinsic DOF's) from as high as 26 dimensions in the case of the human hand.

\section{B. Energy Function}

The first energy function formulation that we propose simply attempts to bring a number of pre-selected contact points on the robotic hand in contact with the object (figure 1). The energy contains two terms: the first one sums the distances between the desired contact points and the object surface while the second one sums the angular differences between the orientation of the surface normals at the contact locations and the closest point on the object. By sampling the palm and all the links of the robotic hand, as in figure 1 , we expect the energy function to be minimized when the 
hand is wrapped around the object generating a large contact area.

In most cases, the resulting hand posture creates an enveloping grasp of the object, especially for complex hand models grasping objects similar in size to the hand. However, there exist cases where the desired contact locations are all very close to the object surface without generating a stable grasp. Furthermore, small objects might be impossible to completely wrap the hand around, and an acceptable minimum of the energy function will not exist. To address this problem we also propose a modified version of the energy function that includes a built-in notion of grasp quality as described below.

While a number of grasp quality metrics have been presented in the literature, our context is somewhat different: we require a metric that can take into account not only existing contacts between the hand and the object, but also potential contacts that can be realized by small changes in the current state. In this sense, the ideal metric would assess the potential of a hand posture, and determine whether the annealing algorithm will search its neighborhood for progressively better states.

One possible quality metric that can be modified according to these requirements is the one described by Ferrari and Canny [15]. In its original form, the process involves building the space of wrenches that can be applied by a grasp (the grasp wrench space, or GWS) by taking the convex hull of the wrenches that can be applied through each contact. If the origin is not contained in this space, the grasp does not have force-closure (F-C) and the quality is 0 . Otherwise, the quality of the grasp is is equal to the distance from the origin to the closest boundary of the GWS. In our implementation, object contacts are replaced by the desired contact locations exemplified in figure 1 . When computing the GWS, we scale the wrenches that can be applied at each desired contact location depending on the distance between the desired contact and the object surface. Thus, if this distance is small, the contact will have a positive contribution to the grasp, and states that bring it closer to the object surface will be rewarded by a higher quality value. If, on the contrary, the desired contact is far from the object, it will not significantly affect the grasp quality measurement.

Once the grasp quality term is computed, it is included in the energy function in negated form, as the annealing algorithm attempts to minimize the energy value. Its contribution biases the search algorithm toward states that not only bring the hand in contact with the object, but also create stable grasping postures. We have found that these formulations work well in practice, as shown in the following sections. However the ideal energy function formulation, which would guarantee stable grasps and eliminate the need to pre-specify desired contact locations on the hand, is the subject of further research.

\section{Simulated Annealing example}

We have implemented the simulated annealing approach using the publicly available GraspIt! simulation engine [16].
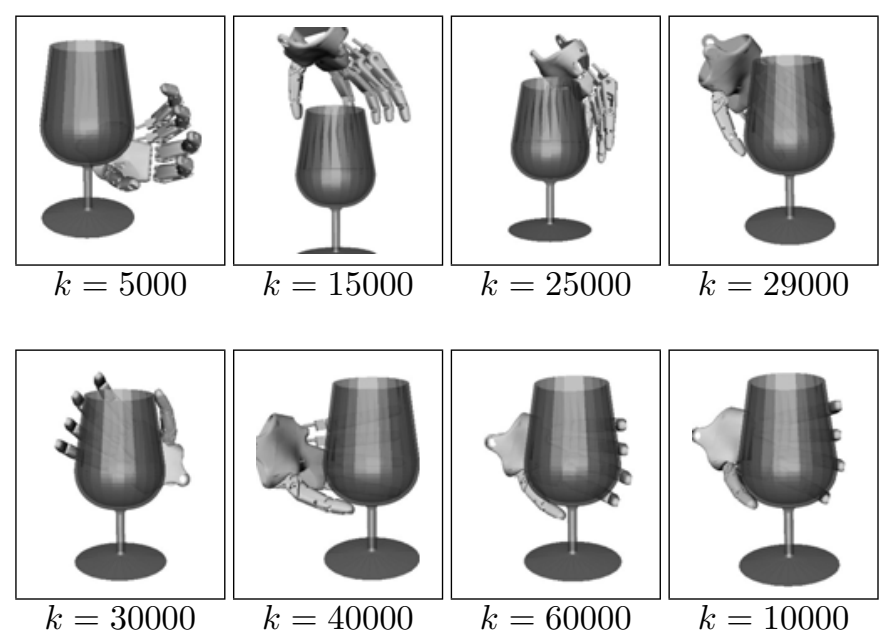

Fig. 2. Simulated annealing example over 100,000 iterations. Each image shows the best state found until iteration $k$.

For each state generated during the annealing schedule, GraspIt! uses forward kinematics to place the robotic hand model in the correct posture and checks for collisions against the object to be grasped as well as other obstacles. If the state is found to be legal, the corresponding energy function is computed and the annealing algorithm continues. This process can be repeatead until a satisfactory energy level has been reached, or a pre-specified number of iterations has been exceeded.

Before presenting an extensive test of this optimization method, involving multiple hand models as well as different objects, we will discuss a typical example, in order to analyze the behavior of the simulated annealing algorithm in more detail. This example involves the Robonaut hand grasping a glass. The optimization was performed over 100,000 iterations, which is the case for all examples shown in this paper. Figure 2 shows the temporary solution (best state found so far) at various points during the optimization. We can observe what is considered typical behavior for a simulated annealing implementation: at first, the search goes through random states, accepting bad positions as well as good positions. As the annealing schedule progresses, the search space is sampled more often in the vicinity of the good states, while bad states are no longer accepted. Finally, in the later stages, the search is confined in a small neighborhood around the best state, which is progressively refined. The total time required for the optimization was 173 seconds, or 1.73 milliseconds / iteration. The most significant amount of computation was spent checking the feasibility of each generated state (i.e. checking for collisions and interpenetrations).

\section{GRASP PLANNING EXAMPLES}

In order to test the effectiveness of our framework, we have applied the eigengrasp planning algorithm using all five previously discussed robotic hand models on a set of six objects. Figure 3 shows the result of the annealing search for each hand-object combination. In order to allow a direct assessment of the planning method through visual inspection 


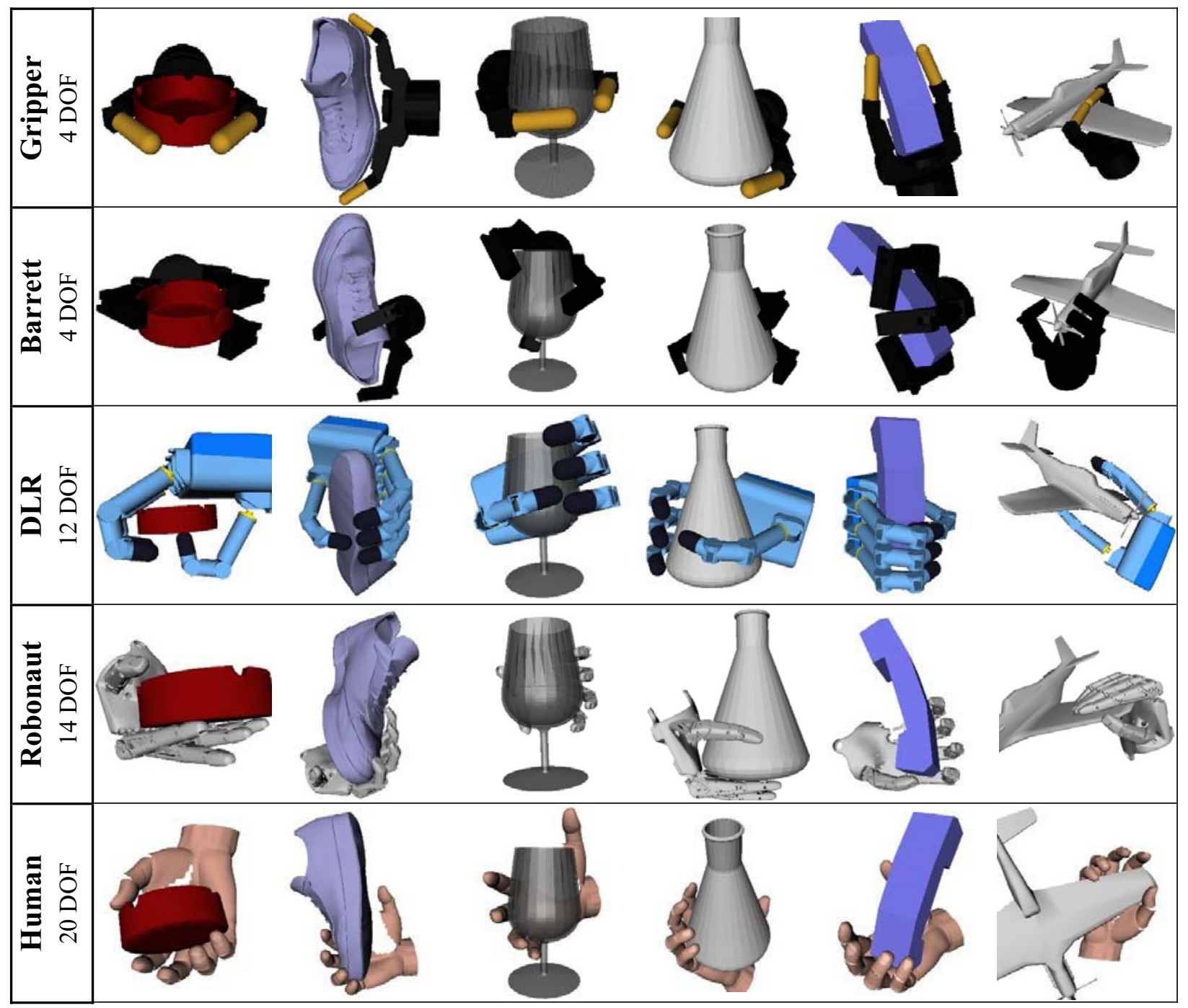

Fig. 3. Eigengrasp planner test using 5 hand models to grasp each of 6 objects

of the results, figure 3 presents the best hand posture found by the planner without any additional refinements. We note that, in most cases, planning in the reduced space spanned by only two eigengrasps does not result in a posture where the robotic hand conforms perfectly to the surface of the object. However, the result is often close enough to such a posture that a stable grasp can be obtained by using simple heuristics. One possible heuristic involves closing each finger until contact with either the object or another finger prevents further motion. This method produces a force-closure grasp in 23 out of the 30 cases presented in figure 3 .

For the results presented in figure 3, we have specified desired contact locations on the entire surface of the robotic palm. However, it is also possible to use only a subset of these. For example, we can choose to use only the fingertip contacts, thus removing the requirement of wrapping the hand around the object. In this case, the grasp quality component of our energy function (section III-B) takes vital importance, as it is generally easy to simply place the fingertips on the object surface without necessarily creating a strong grasp. This approach can be regarded as an attempt to find good manipulation grasps and, especially in the case of robotic hands equipped with human-like fingertips, it produces stable results. Examples are shown in figure 4.a. for the DLR, Robonaut and Human hand models, with the note that all presented grasps have force-closure.

Finally, we have used the method presented in this paper to plan grasps in the presence of obstacles. Figure 4.b. shows a situation in which a table surface prevents the execution of the best grasps, thus forcing the algorithm to find alternative solutions. The only additional cost incurred by the grasp planner is that of collision detection against the obstacle for each newly generated state. Again, all the grasps presented in the image have force-closure.

One object in our test set that requires additional consideration is the toy airplane model. It differs from the rest of the set in the sense that it can not be well approximated using a single convex component, as it is the sum of a number of dominant shapes (fuselage, wings, etc.). The grasp planner that we have presented poses no hard convexity constraint on the object, and it produces a number of convincing grasps on the airplane model. However, an intuitive method to simplify 


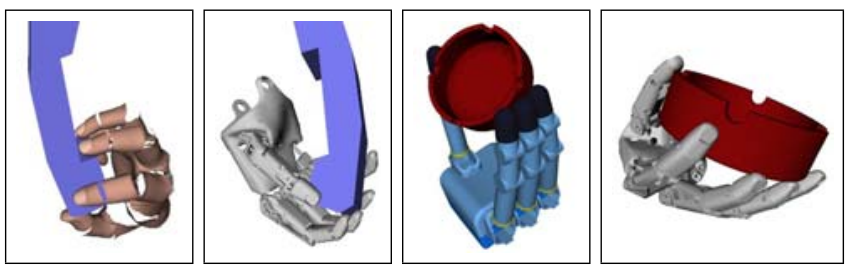

a) Planning results using only fingertip contacts
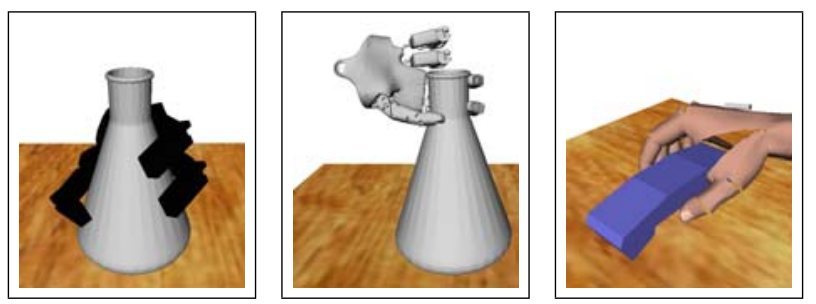

b) Planning results with external obstacles, such as a table surface

Fig. 4. Grasps planning with additional constraints

the search would be to decompose the object into dominant convex sub-parts and plan individually on each of these. In previous work we showed how approximating an object with smooth convex primitives can be used to reduce the complexity of the grasp search space [17]. Psychological research [18] suggests that such "grasping by parts" is similar to the cognitive approach humans use for grasping, and as such we are interested as future work in combining this type of humanlike cognitive modeling with our humanlike hand control.

\section{CONCLUSIONS}

In this paper we have built upon recent results in neuroscience research, which show that human hand control for common grasping tasks mostly takes place in a space of much lower dimensionality than the number of degrees of freedom of the human hand. We have extended this concept for a number of robotic hands, ranging from a simple gripper to highly anthropomorphic models. For each model, we have defined a low dimensional subspace of the degrees of freedom space, determined by a number of basis vectors which we call eigengrasps.

As long as the eigengrasp space provides a good approximation of the hand motion required for a given task, control algorithms can be designed to operate in this space and take advantage of the dimensionality reduction. In the case of human grasping, data collected through user studies has shown that this is indeed the case. In this paper we show that this is also the case for complex robotic hands: we have built an automated grasp planner that can find stable grasps for complex hand models that have traditionally been very difficult to plan for.

The eigengrasp space acts not only to reduce control complexity, but also as an interface between the kinematic structure of the hand and higher-level task planning. Therefore, for a given task, it is possible to use a unified treatment for a number of robotic hand models, even though the kinematic specifications may be significantly different. We have illustrated this concept by using the eigengrasp planner on five robotic hands, with the number of intrinsic DOF's ranging between 4 and 20. The results show that it is indeed possible to apply an identical control algorithm to all of these hand models and obtain consistent results. Furthermore, the eigengrasp planning method we have presented can be used to find both power and manipulation grasps, and can take into account the presence of external obstacles.

While this work has been focused on the task of grasping everyday objects, we believe that eigengrasp-like control synergies can be found for many other domains. Since the published experimental data we draw upon was collected under such assumptions, we found it unjustified to generalize our particular choices of eigengrasps without further analysis. However, the effectiveness of the grasp planning algorithm based on relatively few eigenvectors of hand motion suggests that identifying similar dimensionality reduction strategies for other domains (such as manipulation) will prove a fruitful area of future research.

\section{REFERENCES}

[1] J. R. Napier, "The prehensile movements of the human hand," Journal of Bone and Joint Surgery, vol. 38, pp. 902-913, 1956

[2] M. R. Cutkosky, "On grasp choice, grasp models, and the design of hands for manufacturing tasks," IEEE Transactions on Robotics and Automation, vol. 5, pp. 269-279, 1989.

[3] T. Iberall, "Human prehension and dexterous robot hands," International Journal of Robotics Research, vol. 16, pp. 285-299, 1997.

[4] M. Santello, M. Flanders, and J. F. Soechting, "Postural hand synergies for tool use," Journal of Neuroscience, vol. 18, no. 23, pp. $10105-$ $10115,1998$.

[5] R. A. Peters and O. C. Jenkins, "Uncovering manifold structures in robonaut's sensory-data state space," in IEEE-RAS Intl. Conf. on Humanoid Robots, 2005, pp. 369-374.

[6] A. Tsoli and O. C. Jenkins, "2d subspaces for user-driven robot grasping," in Robotics, Science and Systems Conference: Workshop on Robot Manipulation, 2007.

[7] A. T. Miller, S. Knoop, H. I. Christensen, and P. K. Allen, "Automatic grasp planning using shape primitives," IEEE Intl. Conf. on Robotics and Automation, vol. 2, pp. 1824-1829, 2003.

[8] J. Aleotti and S. Caselli, "Grasp recognition in virtual reality for robot pregrasp planning by demonstration," in IEEE Intl. Conf. on Robotics and Automation, Orlando, FL, May 2006.

[9] Y. Li and N. S. Pollard, "A shape matching algorithm for synthesizing humanlike enveloping grasps," in IEEE-RAS International Conference on Humanoid Robots, Tsukuba, JP, December 2005, pp. 442-449.

[10] K. B. Shimoga, "Robot grasp synthesis algorithms: a survey," Intl. J. of Robotics Research, vol. 15, pp. 230-266, 1996.

[11] A. Bicchi and V. Kumar, "Robotic grasping and contact: A review," IEEE Intl. Conf. on Robotics and Automation, pp. 348-353, 2000.

[12] C. S. Lovchik and M. A. Diftler, "The robonaut hand: A dextrous robot hand for space," in IEEE Intl. Conf. on Robotics and Automation, 1998, pp. 907-912.

[13] J. Butterfass, G. Hirzinger, S. Knoch, and H. Liu, "Dlr's articulated hand, part i: Hard- and software architecture," in IEEE Intl. Conf. on Robotics and Automation, 1998, pp. 2081-2086.

[14] L. Ingber, "Very fast simulated re-annealing," J. Mathl. Comput. Modelling, vol. 12, no. 8, pp. 967-973, December 1989.

[15] C. Ferrari and J. Canny, "Planning optimal grasps," in IEEE Intl. Conf. on Robotics and Automation, 1992, pp. 2290-2295.

[16] A. Miller and P. K. Allen, "Graspit!: A versatile simulator for robotic grasping," IEEE Robotics and Automation Magazine, vol. 11, no. 4, pp. 110-122, December 2004.

[17] C. Goldfeder, P. Allen, C. Lackner, and R. Pelossof, "Grasp planning via decomposition trees," in IEEE Intl. Conf. on Robotics and Automation, Rome, Italy, April 2007.

[18] I. Biederman, "Recognition-by-components: A theory of human image understanding," Psychological Review, vol. 94(2), pp. 115-147, 1987. 